\title{
A food web perspective on large herbivore community limitation
}

\author{
Hervé Fritz, Michel Loreau, Simon Chamaillé-Jammes, Marion Valeix and Jean Clobert \\ H. Fritz (herve.fritz@univ-lyonl.fr) and M. Valeix, CNRS UMR 5558, Laboratoire de Biométrie et Biologie Evolutive, Univ. Claude Bernard \\ - Lyon 1, Bât. Gregor Mendel, 43 Bd du 11 Novembre 1918, FR-69622 Villeurbanne Cedex, France. - M. Loreau, Dept of Biology, McGill \\ Univ., 1205 Ave. Docteur Penfield, Montreal, QC H3A 1B1, Canada. - S. Chamaillé-Jammes, Centre d'Ecologie Fonctionnelle et Evolutive, \\ UMR 5175 CNRS, FR-34293 Montpellier Cedex 5, France. - J. Clobert, Station d'Ecologie Expérimentale du CNRS à Moulis, Laboratoire \\ Evolution er Diversité Biologique, Moulis, FR-09200 Saint-Girons, France.
}

\begin{abstract}
The exceptional diversity of large mammals in African savannas provides an ideal opportunity to explore the relative importance of top-down and bottom-up controls of large terrestrial herbivore communities. Recent work has emphasized the role of herbivore and carnivore body size in shaping these trophic relationships. However, the lack of across-ecosystem comparisons using a common methodology prohibits general conclusions. Here we used published data on primary production, herbivore and carnivore densities and diets to estimate the consumption fluxes between three trophic levels in four African savanna ecosystems. Our food web approach suggests that the body size distribution within and across trophic levels has a strong influence on the strength of top-down control of herbivores by carnivores and on consumption fluxes within ecosystems, as predicted by theoretical food web models. We generalize findings from the Serengeti ecosystem that suggest herbivore species below $150 \mathrm{~kg}$ are more likely to be limited by predation. We also emphasize the key functional role played by the largest species at each trophic level. The abundance of the largest herbivore species largely governs the consumption of primary production in resident communities. Similarly, predator guilds in which the largest carnivore species represent a larger share of carnivore biomass are likely to exert a stronger top-down impact on herbivores. Our study shows how a food web approach allows integrating current knowledge and offers a powerful framework to better understand the functioning of ecosystems.
\end{abstract}

Understanding the relative importance of top-down and bottom-up controls in terrestrial ecosystems is a challenging issue in ecology, in particular in ecosystems dominated by large mammals (Halaj and Wise 2001, Shurin et al. 2002). Although strong trophic cascades may occur when vertebrate endotherm predators are extirpated or introduced (Borer et al. 2005), as exemplified in some Northern American systems (McLaren and Peterson 1994, Ripple and Beschta 2004) and suggested in Australia (Letnic and Koch 2009), the lack of comparative food web analyses of large diverse mammalian communities precludes broad conclusions (Duffy et al. 2007). African savannas are terrestrial ecosystems in which there is still a high diversity of large mammalian herbivore and carnivore species (hereafter large herbivores and large carnivores respectively; Fritz and Loison 2006), contrary to most temperate systems where large predators have been extirpated by humans. This unique feature might make African savannas different from other terrestrial systems regarding the relative importance of bottom-up and topdown processes in limiting large vertebrate communities. The effect of large carnivores on ungulate populations in species-rich communities was brought to attention several decades ago (Smuts 1978). Many population studies, however, have suggested bottom-up control of large herbivores in African savannas (Owen-Smith 1990, Mduma et al. 1999), supported by analyses at the community level showing that large ungulate biomass was directly related to primary production (Coe et al. 1976, Fritz and Duncan 1994). A positive relationship between primary and secondary production is also observed across terrestrial ecosystems (McNaughton et al. 1989). However, recent population studies suggest predation limits some large herbivore populations (Sinclair et al. 2003, Grange et al. 2004, Owen-Smith and Mills 2006).

Population limitation mechanisms may differ between species in a community. In particular, body size is likely to play a major role in setting the relative roles of predation and resource availability at the population level. Models show that herbivore-plant size ratios may influence the strength of trophic cascades (Shurin and Seabloom 2005), and that the distribution of body sizes within communities may determine the relative importance of top-down and 
bottom-up controls in entire food webs (Loeuille and Loreau 2005). A natural experiment of predator removal in an East African savanna recently underlined the role of predation in the regulation of small- and medium-sized ungulate populations (Sinclair et al. 2003), suggesting a potential for strong trophic cascades in this system. In multispecies prey communities, a predator may also strongly regulate its primary prey population if there are secondary prey that provide enough energy to subsidize it in times when primary prey decrease in abundance (Sinclair et al. 2003). Sinclair et al. (2003) also suggested that the diet breath, body size and species diversity of predators may enhance the role of predation in limiting some populations, in line with theoretical and empirical work conducted in widely different systems (Thébault and Loreau 2003, Jiang and Morin 2005).

Hopcraft et al. (2010) recently reviewed the literature on African savannas and offered a general framework to predict the relative contribution of bottom-up and top-down controls on herbivores across environmental gradients. They argued that the functioning of savannas is primarily determined by a) vegetation quality and productivity that directly influence the differential performance of herbivores of different body sizes, and b) how carnivores of different body sizes partition their food niches along a herbivore body size gradient. However, the paucity of across-ecosystem comparisons using common standardized methods creates an important gap in our ability to assess this framework.

Here we develop a food web approach (i.e. a quantification of biomass consumption across species or trophic levels, hereafter referred to as consumption fluxes) to assess the relative importance of top-down and bottom-up processes in controlling ungulate populations in four species-rich African savanna ecosystems. Our approach does not aim to describe the dynamics of the system, as the relative strength of interactions may change over time concordant with changes in the relative abundances of prey and predator species, but to quantify consumption fluxes between species and trophic levels to assess whether predation was likely to limit large herbivore populations and thus to provide insights on the factors governing food web structure. Comparative ecosystem studies have proved useful for investigating the strength of trophic cascades and their possible sources of variation (Halaj and Wise 2001, Shurin et al. 2002, Borer et al. 2005). Here we use ecosystems that show contrasting consumption fluxes between primary producers and the various large herbivore species, as well as diverse predation pressures (Fig. 1). We quantify herbivory and predation in ecosystems with a high biomass of large herbivores, for which data are scarce (Schmitz et al. 2000, Halaj and Wise 2001, Shurin et al. 2002, Borer et al. 2005). By taking the complexity of trophic relationships into account and applying the same methodology to different ecosystems, we aim to better understand the processes regulating trophic cascades, and particularly the role of body size in these processes. We expect resident herbivore communities composed mostly of small species (sensu Sinclair et al. 2003: <150 kg) to be more influenced by top-down processes than those dominated by large herbivores. This should also result in the former showing lower herbivory pressure.

\section{Materials and methods}

\section{Study systems}

The four studied savanna ecosystems - Hwange, Kruger, Ngorongoro and Serengeti - are all protected areas. The four ecosystems differ in soil nutrient availability (sensu Bell 1982): Hwange National Park (Zimbabwe; $19.1^{\circ} \mathrm{S}, 26.6^{\circ} \mathrm{E}$ ) has poor soils, Kruger National Park (South Africa; $24.0^{\circ} \mathrm{S}$, $31.5^{\circ} \mathrm{E}$ ) has medium-poor soils, and Serengeti National Park and Ngorongoro Crater National Park (Tanzania, respectively: $2.5^{\circ} \mathrm{S}, 34.6^{\circ} \mathrm{E}$ and $3.1^{\circ} \mathrm{S}, 35.4^{\circ} \mathrm{E}$ ) have rich soils. We focused on the northern section of the Serengeti and on the Main Camp section of Hwange National Park, for which we had data on carnivore diets. We considered that migratory ungulates are present 6 months in a year in the northern section of the Serengeti (Fryxell 1995).

These systems also correspond to different profiles in the biomass distribution across large herbivore body sizes. Hwange is dominated by megaherbivores (sensu OwenSmith 1988, body weight >1000 kg), mostly elephants Loxodonta africana, the Serengeti and Ngorongoro by medium- to large-sized species $(150-300 \mathrm{~kg})$, while the Kruger is more balanced (Fig. 1). An additional interest of using the Serengeti is that we can compare the results of our food web approach and our subsequent predictions on the relative role of predation with those of an earlier natural predator removal experiment (Sinclair et al. 2003). For this comparison, we used the northern section of the Serengeti and distinguished resident and migratory ungulates (Sinclair et al. 2003) because migration is an efficient anti-predator strategy that has a considerable impact on the trophic fluxes in this ecosystem (Fryxell 1995). Predator species are the same in the four systems, but their abundance and consumption impact on herbivores differ substantially (Fig. 1).

\section{Data and analyses}

We used published data on primary production, herbivore and carnivore densities and diets for the Kruger, Serengeti and Ngorongoro (Kruuk 1972, Schaller 1972, Sinclair and Norton-Griffiths 1979, Homewood and Rodgers 1991, Scholes and Walker 1993, Caro 1994, Sinclair and Arcese 1995, Boone et al. 2000, du Toit et al. 2003, Estes et al. 2006), and unpublished data for Hwange (Drouet-Hoguet, Rasmussen, Fritz, Loveridge and Macdonald pers. comm.).

We converted population density into biomass for the various herbivore species using body mass data from Coe et al. (1976), which were completed by Cumming and Cumming (2003). African herbivore communities cover a wide range of body sizes, ranging from steenbok Raphicerus campestris (female adult body mass $=11 \mathrm{~kg}$ ) to elephant (female adult body mass $=2275 \mathrm{~kg}$ ). Body size determines many physiological and ecological attributes (Peters 1983). In particular, metabolic needs scale allometrically with body mass, hence we estimated herbivore dry matter daily food intake from allometric studies (Illius and Gordon 1992). Besides, age at first reproduction and fecundity scale inversely with body size, hence we used these life history 

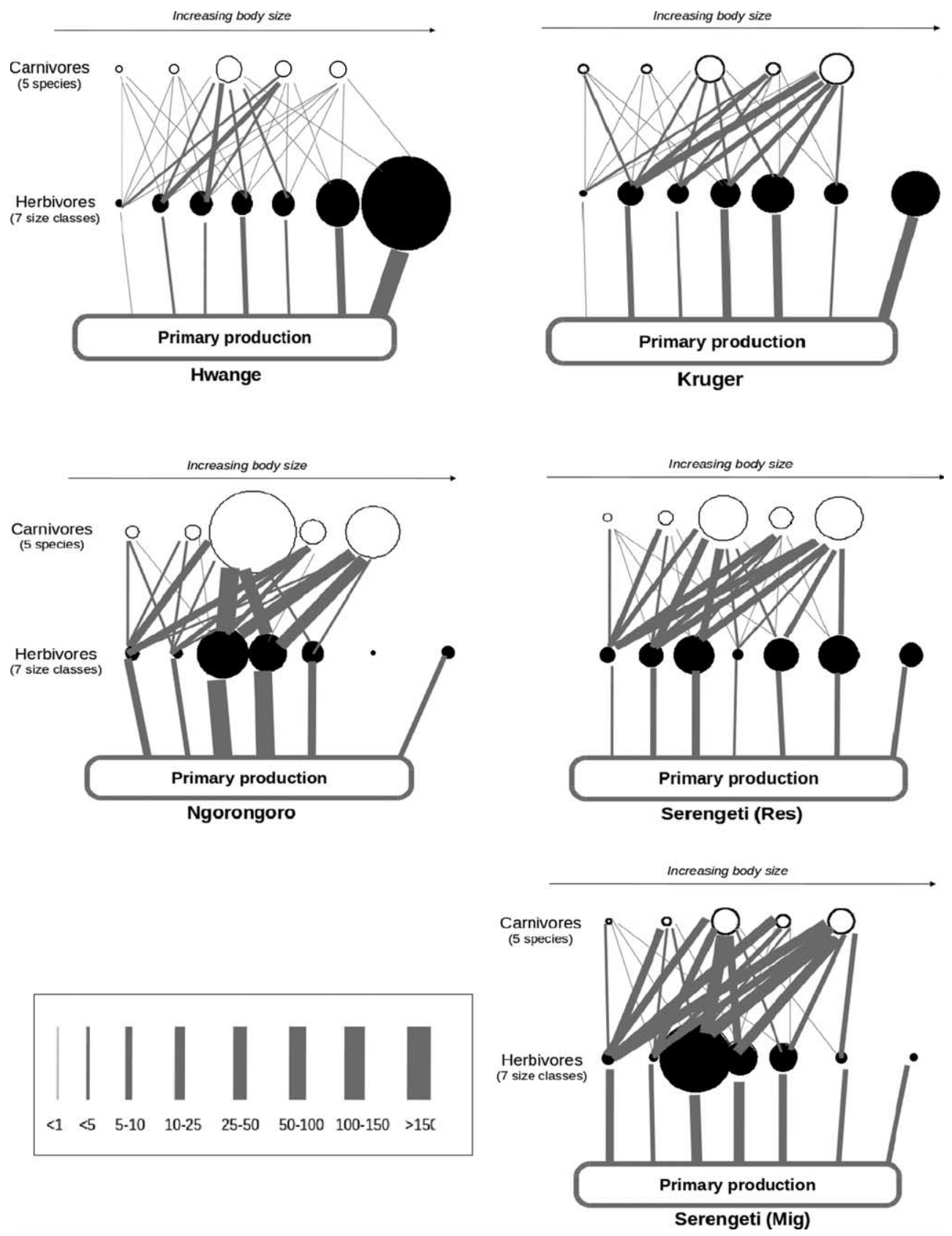

Figure 1. Reconstructed food webs for the four savanna ecosystems, distinguishing between the migratory and resident system in the Serengeti. The same five species of carnivores are present in all ecosystems, i.e. from left to right, the African wild dog Lycaon pictus ( 25 $\mathrm{kg}$ ), the cheetah Acinonyx jubatus (45 kg), the hyaena Crocuta crocuta $(60 \mathrm{~kg})$, the leopard Panthera pardus ( $40 \mathrm{~kg})$, and the lion Panthera leo $(145 \mathrm{~kg})$. In this figure leopard and hyaena are swapped along the body size gradient for clarity. The herbivores size classes are similar to those in Fig. 2: 10-25, 40-70, 90-140, 160-250, 300-500, 700-900, >1500 kg. Circle diameters represent the biomass $\left(\mathrm{kg} 100 \mathrm{~km}^{-2}\right)$ of carnivore species and herbivore body mass classes. The values were transformed for graphical convenience $(0.3 \sqrt{ }$ for carnivores and $0.5 \mathrm{~V}$ for herbivores). Arrow widths are proportional to prey-predator consumption fluxes. For herbivores, units are in kg of dry matter $\mathrm{d}^{-1} \mathrm{~km}^{-2}$, for carnivores units are in $\mathrm{kg}$ of fresh weight $\mathrm{d}^{-1} 100 \mathrm{~km}^{-2}$.

traits or Sinclair's (1996) allometric equation to calculate the maximum annual rate of population increase for each herbivore species assuming no mortality.

Daily food requirements for each carnivore species (in $\mathrm{kg}$ of fresh meat) was estimated using published values from a recent review (Carbone et al. 2007). We used ecosystem-specific data on carnivore diets to estimate at each system the contribution of each prey species to the annual consumption of each carnivore species. For each herbivore species, we then converted the biomass consumed by all carnivores in a year into a number of individuals consumed, and calculated annual predation rate as the proportion of individuals consumed by carnivores in the population. Lastly, we estimated 
population growth rates of herbivores after predation as the difference between the maximum annual rate of population increase and the annual predation rate.

To estimate the aggregate consumption fluxes between trophic levels in each ecosystem, we calculated the fraction of primary production consumed by all large herbivores, distinguishing between three body size classes (mesoherbivores $<150 \mathrm{~kg}$, mesoherbivores $\geq 150 \mathrm{~kg}$, and megaherbivores), and the fraction of herbivore biomass consumed by all carnivores. Statistical analyses of annual predation rates used log-transformed data to conform to the requirements of parametric statistics.

\section{Results}

Consumption fluxes within the studied ecosystems are presented in Fig. 1. Annual predation rate decreased significantly with increasing prey body size $\left(\mathrm{F}_{1,44}=\right.$ $10.95, \mathrm{p}=0.002, \mathrm{r}^{2}=0.60$ ), i.e. smaller-sized herbivores were more heavily impacted by predation (Fig. 2), and there was no significant system effect $\left(\mathrm{F}_{4,44}=0.67, \mathrm{p}=0.62\right)$. The expected population growth rates of small- to mediumsized herbivores, after accounting for predation, were negative (Fig. 2). Based on these estimates, predators were likely, not only to limit, but even to reduce small herbivore populations.

The Serengeti resident system and the Kruger system both had a greater consumption of herbivores by carnivores than of plants by herbivores, whereas the reverse was true for Ngorongoro and Hwange (Fig. 3). The predator:prey biomass ratio (excluding elephants) was similar between Hwange and Ngorongoro (0.008 and 0.01, respectively), and was at least twice lower than in the Serengeti resident system and the Kruger (0.03 and 0.02 , respectively).

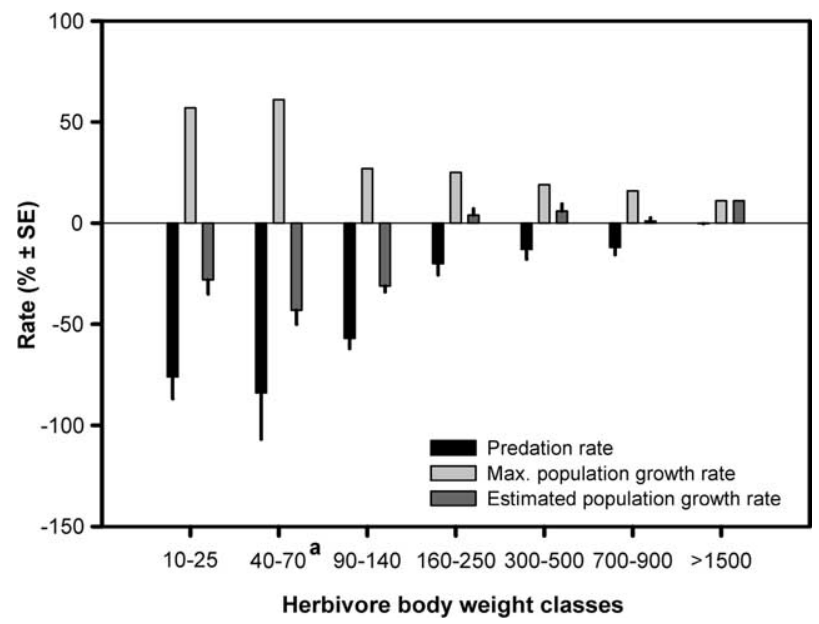

Figure 2. Maximum annual rates of population increase, estimated annual predation rates averaged across ecosystems (mean \pm $\mathrm{SD}$ ), and estimated annual population growth rates averaged across ecosystems (mean $\pm \mathrm{SD}$ ) after accounting for predation for the seven body mass classes of herbivores. Species with body mass below $150 \mathrm{~kg}$ appear more likely to be limited by predation, as they exhibited estimated negative or zero growth. ${ }^{\mathrm{a}}$ Includes Suids, which have much higher maximum population rate of increase $(>100 \%)$ but also much higher observed annual predation rate $(>80 \%)$.

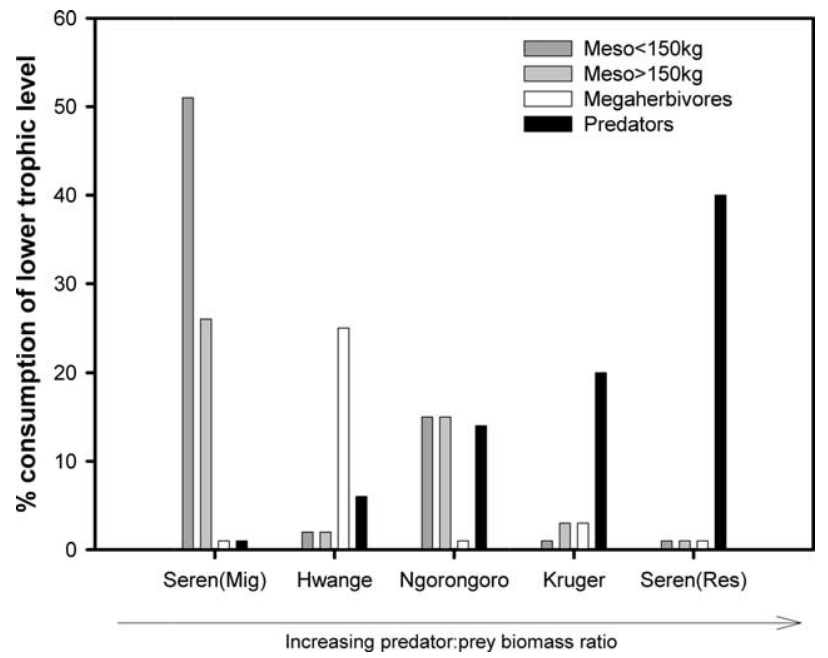

Figure 3. Percentage of the lower trophic level consumed yearly by carnivores and three herbivore body size classes, calculated as proportions of total herbivore biomass consumed for carnivores, and proportions of total primary production consumed for herbivores. Herbivores are grouped in three classes instead of seven, both for clarity and because the first two classes and the last two classes in Fig. 1 and 2 showed similar patterns. The systems are ordered from right to left along a gradient of increasing predator:prey biomass ratio. The resident systems showed higher predation level, in particular where lions make most of the carnivore biomass (Kruger and Serengeti resident). The highest herbivory levels were calculated in resident system largely dominated by megaherbivores (Hwange) or in the Serengeti migratory system.

Hwange was the only system where the contribution of elephants changed the predator:prey ratio significantly; its predator:prey ratio including elephants $(0.002)$ was close to the lowest ratio observed in the Serengeti migratory system (0.001).

The overall impact of predation on prey in each system depended on its predator:prey biomass ratio since the rank correlation between the average predation rate and the predator:prey biomass ratio was significant $\left(n=5, r_{s}=0.9\right.$ and $r_{s}=1$, both $\mathrm{p}<0.05$, with and without elephants in the predator:prey ratio calculation, respectively). This conclusion did not hold for the predator:prey density ratio (both $p>0.05$ ), which suggests that the body mass of the dominant carnivores played a greater role than their density. Systems where lions Panthera leo comprised most of the carnivore biomass exhibited greater predation pressure on most herbivore body size classes (Fig. 4), and were characterised by greater predation pressure on large herbivores between 700 and $900 \mathrm{~kg}$, which had an estimated growth rate close to zero (Fig. 4).

\section{Discussion}

Our food web approach suggests that predation could be limiting in most systems for small- and medium-sized ungulate species, thus supporting and extending previous findings by Sinclair et al. (2003) (although in Kruger the high abundance of lions also subjects large preys to high predation pressure - see also Owen-Smith and Mills 2008). 


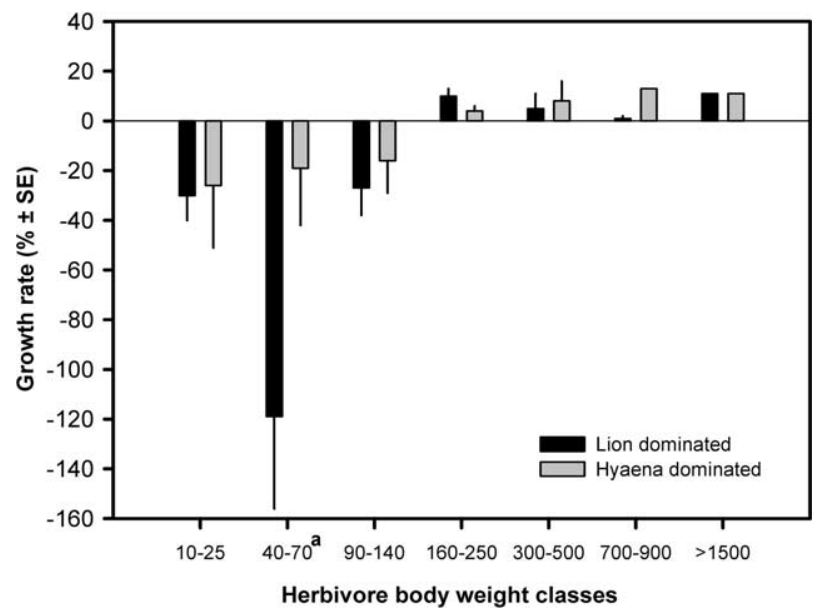

Figure 4. Estimated population growth rates for the seven body mass classes of herbivores in ecosystems where lions comprised most of the carnivore biomass (Kruger and Serengeti) and those where hyaena comprised most of the carnivore biomass (Hwange and Ngorongoro). Estimated population growth rates were first calculated for each species in each system, then averaged over lion dominated and hyaena dominated systems, and finally averaged per herbivore body mass class. The systems dominated by lions appear to have lower calculated growth rates, implying a greater predation pressure, from the smaller to even the very large herbivore species. ${ }^{a}$ Includes Suids, which have much higher observed annual predation rate $(>80 \%)$.

Our estimates predict that some herbivore species should disappear quickly from overexploitation by carnivores. This is unrealistic and could be explained by a potential underestimation bias in herbivore census data (sometimes by up to $30 \%$ : Jachmann 2002). Additionally, our estimates of annual predation rates were constant and came from instantaneous estimates even though predation rates are likely to decrease as prey become scarcer. Nevertheless, the estimated levels of predation are large enough to suggest that predation strongly regulates populations of herbivore species below $150 \mathrm{~kg}$, since for many species our estimates predict that virtually all newborn individuals could be consumed by predators. If predation were entirely compensatory, that is, predated animals would die anyhow, then predation would not act as a limiting process (Owen-Smith and Mills 2008). This assertion, however, appears highly unlikely given the high predation levels estimated here.

Our results also suggest that the relative importance of top-down and bottom-up processes may vary greatly between systems, even though species composition was very similar, suggesting a crucial role of the relative abundance of the various body size classes. The Serengeti resident system and the Kruger system both showed greater levels of consumption of herbivores by carnivores than of plants by herbivores, whereas the reverse was true in Ngorongoro and Hwange. The main feature that distinguishes these two groups of ecosystems is not their overall abundance of predators, since Ngorongoro has the highest and Hwange the lowest abundance of predators, but their different predator:prey biomass ratios, with higher ratios associated with higher herbivore consumption by carnivores. Also, the largest carnivore, lion, makes up most of the predator biomass in the Serengeti and Kruger whereas the spotted hyaena Crocuta crocuta makes up most of the predator biomass in Hwange and Ngorongoro. Interestingly, our comparative data suggest the prevalence of size-nested predation (sensu Hopcraft et al. 2010: the prey base of small predators is nested within that of larger predators) within the large African predator guild. In this situation an increase in the relative abundance of the largest carnivore (lions) increases predation pressure on the larger herbivores without freeing smaller herbivores from predation.

In systems with resident herbivores, the total consumption of primary production was $<40 \%$, which is consistent with existing studies on other terrestrial ecosystems (Halaj and Wise 2001). The largest effect on primary production occurred in the system dominated by megaherbivores, which were virtually free of predators. A singular exception is the Serengeti where medium-sized grazers avoid predation by migrating (Fryxell 1995) and consume almost all of the available primary production. Theoretical models predict that this is conducive to grazing optimization (de Mazancourt et al. 1998), and the Serengeti is one of the best studied examples of this phenomenon (McNaughton 1985). Resident herbivores, however, consume only a minor proportion of primary production, possibly being held in check by predator populations boosted by the seasonal influx of migratory prey.

The main functional differences between the ecosystems considered in our study were their different predator:prey biomass ratios and different body size distributions within and across trophic levels. The distribution of herbivore body sizes within an ecosystem depends on the abiotic template, particularly as small herbivores are strongly constrained by food quality (Illius and Gordon 1992), which may explain why megaherbivores dominate poor-soil ecosystems. The interplay between environmental gradients, herbivore body size, and predation pressure seems consistent with the conceptual framework proposed by Hopcraft et al. (2010). Here we provide the comparative analysis called for by these authors, and demonstrate the strength of a food web approach to the study of large vertebrate communities. Our approach supports the importance of size-structured predation on consumption fluxes across trophic levels in savanna ecosystems. Although predation rates were probably overestimated, they suggest top-down limitation of small- and medium-sized herbivores. Body size diversity may thus be a key component of ecosystem functioning, both within trophic levels (horizontal diversity) and between trophic levels (vertical diversity) (Duffy et al. 2007).

Our results also suggest that the massive decline of very large herbivores (Surovell et al. 2005) as well as the persecution of large predators by humans (Berger 1999) may have drastic effects on the dynamics and functioning of terrestrial ecosystems, similar to the simpler predator-prey system in North America (Ripple and Beschta 2004). Thus, it is likely that temperate terrestrial ecosystems that maintained a large diversity of predators before their extirpation, function today very differently than in the past (Andersen et al. 2006). Future study of these ecosystems could benefit from the food web approach presented here, which integrates current knowledge and offers a powerful comparative framework. 
Acknowledgements - We thank the Director General of the Zimbabwe Parks and Wildlife Management Authority for providing the opportunity to carry out this research in Hwange National Park. This research was carried out within the HERD program (Hwange Environmental Research Development) funded by the French Ministry of Foreign Affairs, the French Embassy in Zimbabwe, the CIRAD and the CNRS, and within the BioFun and FEAR projects funded by the French National Research Agency (respectively ANR-05-BDIV-013-01 and ANR-08-BLAN0022). ML acknowledges a Discovery grant of the Natural Sciences and Engineering Research Council of Canada. We thank N. Owen-Smith, P. Duncan, I. J. Gordon, A. R. E. Sinclair, J.-M. Gaillard and G. Hopcraft for their useful comments on earlier versions of the manuscript.

\section{References}

Andersen, R. et al. 2006. The future role of large carnivores in terrestrial trophic interactions: the northern temperate view. - In: Danell, K. et al. (eds), Large herbivore ecology, ecosystem dynamics and conservation. Cambridge Univ. Press, pp. 413448.

Bell, R. H. V. 1982. The effect of soil nutrient availability on the community structure in African ecosystems. - In: Huntley, B. J. and Walker, B. H. (eds), Ecology of tropical savannas. Springer, pp. 193-216.

Berger, J. 1999. Anthropogenic extinction of top carnivores and interspecific animal behaviour: implications of the rapid decoupling of a web involving wolves, bears, moose and ravens. - Proc. R. Soc. B 266: 2261-2267.

Boone, R. B. et al. 2000. Addressing management questions for Ngorongoro Conservation Area, Tanzania, using the Savanna modelling system. - Afr. J. Ecol. 40: 138-150.

Borer, E. T. et al. 2005. What determines the strength of a trophic cascade? - Ecology 86: 528-537.

Carbone, C. et al. 2007. The costs of carnivory. - PLoS Biol. 5: 363-368.

Caro, T. M. 1994. Cheetahs of the Serengeti plains: group living in an asocial species. - Univ. Chicago Press.

Coe, M. J. et al. 1976. Biomass and production of large African herbivores in relation to rainfall and primary production. - Oecologia 22: 341-354.

Cumming, D. H. M. and Cumming, C. S. 2003. Ungulate community structure and ecological processes: body size, hoof area and trampling in African Savannas. - Oecologia 134: 560-568.

de Mazancourt, C. et al. 1998. Grazing optimization and nutrient cycling: when do herbivores enhance plant production? - Ecology 79: 2242-2252.

du Toit, J. T. et al. 2003. The Kruger experience: ecology and management of savanna heterogeneity. - Island Press.

Duffy, J. E. et al. 2007. The functional role of biodiversity in ecosystems: incorporating trophic complexity. - Ecol. Lett. 10: $1-17$.

Estes, R. D. et al. 2006. Downward trends in Ngorongoro Crater ungulate populations 1986-2005: conservation concerns and need for ecological research. - Biol. Conserv. 131: 106-120.

Fritz, H. and Duncan, P. 1994. On the carrying capacity for large ungulates of African savanna ecosystems. - Proc. R. Soc. B 256: 77-82.

Fritz, H. and Loison, A. 2006. Large herbivores across biomes. - In: Danell, K. et al. (eds), Large herbivore ecology, ecosystem dynamics and conservation. Cambridge Univ. Press, pp. 1949.

Fryxell, J. M. 1995. Aggregation and migration by grazing ungulates in relation to resources and predators. - In: Sinclair, A. R. E. and Arcese, P. (eds), Serengeti II: dynamics, management, and conservation of an ecosystem. Univ. Chicago Press, pp. 257-273.

Grange, S. et al. 2004. What limits the Serengeti zebra population? - Oecologia 140: 523-532.

Halaj, J. and Wise, D. H. 2001. Terrestrial trophic cascades: how much do they trickle? - Am. Nat. 157: 262-281.

Homewood, K. M. and Rodgers, W. A. 1991. Maasailand ecology: pastoral development and wildlife conservation in Ngorongoro, Tanzania. - Cambridge Univ. Press.

Hopcraft, J. G. C. et al. 2010. Herbivore, resources and risks: alternating regulation along primary environmental gradients in savannas. - Trends Ecol. Evol. 25: 119-128.

Illius, A. W. and Gordon, I. J. 1992. Modelling the nutritional ecology of ungulate herbivores: evolution of body size and competitive interactions. - Oecologia 89: 428-434.

Jachmann, H. 2002. Comparison of aerial counts with ground counts for large African herbivores. - J. Appl. Ecol. 39: 841-852.

Jiang, L. and Morin, P. J. 2005. Predator diet breadth influences the relative importance of bottom-up and top-down control of prey biomass and diversity. - Am. Nat. 165: 350-363.

Kruuk, H. 1972. The spotted hyena: a study of predation and social behaviour. - Univ. Chicago Press.

Letnic, M. and Koch, F. 2009. Are dingoes a trophic regulator in arid Australia? A comparison of mammal communities on either side of the dingo fence. - Austral Ecol. 35: 167-175.

Loeuille, N. and Loreau, M. 2005. Evolutionary emergence of size-structured food webs. - Proc. Natl Acad. Sci. USA 102: 5761-5766.

McLaren, B. E. and Peterson, R. O. 1994. Wolves, moose, and tree rings on Isle Royale. - Science 266: 1555-1558.

McNaughton, S. J. 1985. Ecology of a grazing ecosystem: the Serengeti. - Ecol. Monogr. 55: 259-294.

McNaughton, S. J. et al. 1989. Ecosystem-level patterns of primary productivity and herbivory in terrestrial habitats. - Nature 341: 142-144.

Mduma, S. A. et al. 1999. Food regulates the Serengeti wildebeest: a 40-year record. - J. Anim. Ecol. 68: 1101-1122.

Owen-Smith, N. 1988. Megaherbivores. The influence of very large body size on ecology. - Cambridge Univ. Press.

Owen-Smith, N. 1990. Demography of a large herbivore, the greater kudu Tragelaphus strepsiceros, in relation to rainfall. - J. Anim. Ecol. 59: 893-913.

Owen-Smith, N. and Mills, M. G. L. 2006. Manifold interactive influence on the population dynamics of a multispecies ungulate assemblage. - Ecol. Monogr. 76: 73-92.

Owen-Smith, N. and Mills, M. G. L. 2008. Predator-prey size relationships in an African large-mammal food web. - J. Anim. Ecol. 77: 173-183.

Peters, R. H. 1983. The ecological implications of body size. - Cambridge Univ. Press.

Ripple, W. J. and Beschta, R. L. 2004. Wolves and the ecology of fear: can predation risk structure ecosystems? - BioScience 54: 755-766.

Schaller, G. B. 1972. The Serengeti lion: a study of predator-prey relations. - Univ. Chicago Press.

Schmitz, O. J. et al. 2000. Trophic cascades in terrestrial ecosystems: a review of the effects of carnivore removals on plants. - Am. Nat. 155: 141-153.

Scholes, R. J. and Walker, B. H. 1993. An African savanna. Synthesis of the Nylsvley study. - Cambridge Univ. Press.

Shurin, J. B. and Seabloom, E. W. 2005. The strength of trophic cascades across ecosystems: predictions from allometry and energetics. - J. Anim. Ecol. 74: 1029-1038.

Shurin, J. B. et al. 2002. A cross-ecosystem comparison of the strength of trophic cascades. - Ecol. Lett. 5: 785-791.

Sinclair, A. R. E. 1996. Mammal populations: fluctuation, regulation, life history theory and their implications for 
conservation. - In: Floyd, R. B. et al. (eds), Frontiers of population ecology. CSIRO Publ., pp. 127-154.

Sinclair, A. R. E. and Norton-Griffiths, M. 1979. Serengeti: dynamics of an ecosystem. - Univ. Chicago Press.

Sinclair, A. R. E. and Arcese, P. 1995. Serengeti II: dynamics, management and conservation of an ecosystem. - Univ. Chicago Press.

Sinclair, A. R. E. et al. 2003. Patterns of predation in a diverse predator-prey system. - Nature 425: 288-290.
Smuts, G. L. 1978. Interrelations between predators, prey, and their environment. - BioScience 28: 316-320.

Surovell, T. et al. 2005. Global archaeological evidence for proboscidean overkill. - Proc. Natl Acad. Sci. USA 102: 6231-6236.

Thébault, E. and Loreau, M. 2003. Food-web constraints on biodiversity-ecosystem functioning relationships. - Proc. Natl Acad. Sci. USA 100: 14949-14954. 